\title{
SPECTROPOLARIMETRY OF COMET HALLEY
}

\author{
N. VISVANATHAN, Z.MEGLICK \\ MOUNT STROMLO AND SIDING SPRING OBSERVATORIES \\ D.T. WICKRAMASINGHE \\ DEPARTMENT OF MATHEMATICS \\ AUSTRALIAN NATIONAL UNIVERSITY
}

\begin{abstract}
Spectropolarimetric observations from 3800 to $7000 \AA$ were obtained for the nucleus of comet Halley for nine nights during 1985-86. The observations were spaced over phase angle of 2 to $66^{\circ}$. The continuum polarization without molecular-line contamination as well as the polarization of the molecular lines were evaluated. The plot of polarization versus the phase angle shows small negative polarization for angles less than $20^{\circ}$. The highest polarization measured for phase angle $\leq 66^{\circ}$ is $19.6 \%$.The lowest polarization of $-2.0 \%$ has been measured at the phase angle $\sim 10^{\circ}$. The variation of polarization with phase angle is nearly linear in the range 30 to $60^{\circ}$ giving a slope of $0.4316 \%$ per degree. There is small dependence of polarization with the wavelength in the region 3800 to $6500 \AA$. The polarization of the molecular bands $C N, C_{3}, C_{2}(0,0)$ and $C_{2}(1,0)$ have also been evaluated for the spectra of 15 th December 1985, 18th and 19th March 1986 and 8th April 1986.
\end{abstract}

\section{Introduction}

Comets are important astronomical objects as they are probably the most primitive objects in the solar system and have undergone little or no chemical change after their formation from the interstellar matter.Hence there is great interest in understanding the physical and chemical properties of the cometary dust - grains which may throw light into the origin and history of the interplanetary and interstellar dust. The appearance of the bright comet Halley in 1985-86 provided an unique opportunity to study the properties of the cometary grains.

Photometry and polarimetry are the two traditional techniques used to study the comets. The analysis of the degree of polarization of the solar light scattered by cometary dust with wavelength and phase angle allows in principle to determine the optical properties such as albedo, size, coarseness and composition of the grains.In the year 1985 and 1986 comet Halley has been observed for polarization at optical wavelengths by many observers (Dollfus et al. 1988) and references there in). These observations have been obtained through broad and narrow band filters.

In this paper we report the spectro-polarimetric observations of the nucleus region of the comet Halley for nine nights during $85-86$ at a resolution of $6 \AA$ covering the region 3800 to $6500 \AA$. Unlike broad - band filter polarimetry the spectropolarimetry enables us to determine the continuum polarization without molecular line contamination. In addition the comet spectra could be used to derive the wavelength distribution of the continuum as well as the intensities of the emission lines.

\section{Observations}

Observations of the nucleus of the comet Halley were made on nine nights in 1985 and 1986 during which the phase angle of the comet varied from 2 to $66^{\circ}$. Table 1 contains the date of observation, Julian date, distance between the comet and the earth $\Delta$, the distance between the sun and the comet $R$, the size of the aperture projected on to the comet $S$, the distance between the two apertures $D$, the direction of the plane of scattering $\psi$ and the phase angle B. All observations were made with the spectropolarimeter (McLean et al 1984) in combination with the RGO spectrograph + IPCS detector at the Cassegrain focus of AAT in Sidingspring. The 250-line grating was used in combination with $25 \mathrm{~cm}$ camera with a resolution of $6 \AA$. The spectral region from 3800 to $7000 \AA$ was covered with 2000 pixel format. Two apertures of $1.34 \times 2.68$ arcsec with a separation of 22.8 arcsec were used. The nucleus of the comet was centered in one of the apertures.Observations were made altemately at $\mathrm{Q}$ and $U$ polarimetry modes. The sky was observed

\section{5}

A.C. Levasseur-Regourd and H. Hasegawa (eds.), Origin and Evolution of Interplanetary Dust, 245-248. C 1991 Kluwer Academic Publishers, Printed in Japan. 
TABLE 1. Details of the observations of the comet Halley.

\begin{tabular}{|l|l|l|l|}
\hline Date & 18 Oct 1965 & 12 Kov 1985 & 17 Nov 1985 \\
\hline Julian Date (day) & $2446356.74 \pm 0.02$ & $2446381.69 \pm 0.04$ & $2446366.69 \pm 0.02$ \\
$\Delta(\mathrm{AU})$ & $1.4907 \pm 0.0009$ & $0.7961 \pm 0.0007$ & $0.6979 \pm 0.0002$ \\
$R(A U)$ & $2.1071 \pm 0.0002$ & $1.7579 \pm 0.0004$ & $1.6879 \pm 0.0002$ \\
$S_{A}(\mathrm{~km})$ & $1446.7 \pm 0.2$ & $773.7 \pm 0.7$ & $678.3 \pm 0.2$ \\
$D_{A}(\mathrm{~km})$ & $24650 \pm 15$ & $13165 \pm 12$ & $11541 \pm 4$ \\
$\Psi$ & $91^{\circ} 7^{\prime} 17^{\prime \prime} \pm 8^{\prime \prime}$ & $75^{\circ} 25^{\prime} \pm 4^{\prime}$ & $70^{\circ} \pm 3^{\circ}$ \\
$\beta$ & $25^{\circ} 16^{\prime} 29^{\prime \prime} \pm 9^{\prime \prime}$ & $9^{\circ} 56^{\prime} \pm 4^{\prime}$ & $2^{\circ} 7^{\prime} \pm 2^{\prime}$ \\
\hline
\end{tabular}

\begin{tabular}{|l|l|l|l|}
\hline Date & 15 Dec 1985 & 18 Mar 1986 & 19 Mar 1986 \\
\hline Julian Date (day) & $2446414.46 \pm 0.03$ & $2446507.75 \pm 0.04$ & $2446506.75 \pm 0.03$ \\
$\Delta(\mathrm{AU})$ & $0.8192 \pm 0.0007$ & $0.8546 \pm 0.0007$ & $0.8327 \pm 0.0009$ \\
$R(\mathrm{AU})$ & $1.2704 \pm 0.0004$ & $0.9648 \pm 0.0007$ & $0.9826 \pm 0.0003$ \\
$S_{A}(\mathrm{~km})$ & $796.2 \pm 0.7$ & $630.6 \pm 0.4$ & $809 \pm 1$ \\
$D_{A}(\mathrm{~km})$ & $13546 \pm 11$ & $14135 \pm 11$ & $13769 \pm 15$ \\
$\Psi$ & $66^{\circ} 36^{\prime} 30^{\prime \prime} \pm 25^{\prime \prime}$ & $77^{\circ} 31^{\prime} 4^{\prime \prime} \pm 16^{\prime \prime}$ & $77^{\circ} 41^{\prime} 9^{\prime \prime} \pm 22^{\prime \prime}$ \\
$\beta$ & $50^{\circ} 46^{\prime} \pm 2^{\prime}$ & $65^{\circ} 57^{\prime} 48^{\prime \prime} \pm 28^{\prime \prime}$ & $66^{\circ} 6^{\prime} 39^{\prime \prime} \pm 3^{\prime \prime}$ \\
\hline
\end{tabular}

\begin{tabular}{|l|l|l|l|}
\hline Date & 8 Apr 1966 & 12 Apr 1986 & 15 May 1986 \\
\hline Julian Date (day) & $2446526.75 \pm 0.03$ & $2446532.64 \pm 0.03$ & $2446566.40 \pm 0.03$ \\
$\Delta($ AU) & $0.4270 \pm 0.0003$ & $0.4198 \pm 0.0001$ & $1.2367 \pm 0.0008$ \\
$R($ AU) & $1.2927 \pm 0.0003$ & $1.3516 \pm 0.0003$ & $1.6383 \pm 0.0004$ \\
$S_{A}(\mathrm{~km})$ & $415.0 \pm 0.3$ & $407.96 \pm 0.04$ & $1201 \pm 1$ \\
$D_{\Lambda}(\mathrm{km})$ & $7061 \pm 5$ & $6941 \pm 1$ & $20450 \pm 14$ \\
$\Psi$ & $120^{\circ} 52^{\prime} \pm 12^{\prime}$ & $153^{\circ} 53^{\prime} \pm 15^{\prime}$ & $106^{\circ} 20^{\prime} 37^{\prime \prime} \pm 45^{\prime \prime}$ \\
$\beta$ & $39^{\circ} 55^{\prime} \pm 5^{\prime}$ & $26^{\circ} 44^{\prime} \pm 4^{\prime}$ & $31^{\circ} 24^{\prime} 0^{\prime \prime} \pm 1^{\prime \prime}$ \\
\hline
\end{tabular}

separately $3^{\circ}$ away from the nucleus. The exposures for each night varied from 1 to 2 hrs.Each night a solar spectral - type star was observed through the same instrument set - up. The polarization standards HD160529 and $\mathrm{HD} 183143$ and an unpolarized star were also observed to check the performance of the equipment (Visvanathan 1965). The polarization efficiency at each pixel was evaluated by observing $100 \%$ polarised light with the same set-up.The Stokes parameters $Q$ and $U$ were determined and the polarization was computed for each pixel. The wavelength calibration of the spectrum was achieved through the comparison arc spectrum.

\section{Polarization}

The computed polarization $P$ and the position angle of the electric vector $\theta$ as a function of wavelength for the nucleus of the comet observed on 18th March 1986 are shown in Figure 1a and 1b. The spectrum of the nucleus derived from the polarization data was divided by the spectrum of the solar spectral-type star HD186189 and is shown in Figure 1c. The $1 \sigma$ error points are indicated as dotted lines. The molecular emission bands dominate the spectrum in the region from $3800-5600 \AA$. Only regions with width of $\sim 100 \AA$ around $\lambda 3800,4400,4800,5200,6200,6800 \AA$ are mostly free of emission bands. Polarization reaches maximum at these wavebands. Further it can be seen that the molecular bands are also polarized but much less than the continuum. The angle $\theta\left(167^{\circ}\right)$ is nearly constant with wavelength and as expected, is perpendicular to the direction of the plane of scattering, which is $77^{\circ}$ on $18 \mathrm{th}$ March 86 . Small deviations ( $\sim 10^{\circ}$ ) from $167^{\circ}$ is seen in the case of the polarized molecular bands. The polarization of the continuum at $\lambda$ $3800,4400,4800,5200,6200,6800 \AA$ have been evaluated for 15 th Dec 85, 18th Mar 86, 19th Mar 86,and 8 th Apr 86 and plotted in Fig. 3. The error in $P$ is $\sim 0.5 \%$ for values at phase angles $<40^{\circ}$ and $-0.2 \%$ for values at phase angles at $>40^{\circ}$. The wavelength dependence of polarization in the spectral region $\lambda 3800$. $6800 \AA$ is nearly flat at phase angles $<40^{\circ}$. At larger phase angles $P$ is lower at UV with a small increase at higher wavelengths. The data by other observers (Dollfus et al. 1988) indicate a similar wavelength dependence. It is interesting that our results which represents the region of $\sim 2$ seconds of arc around the 

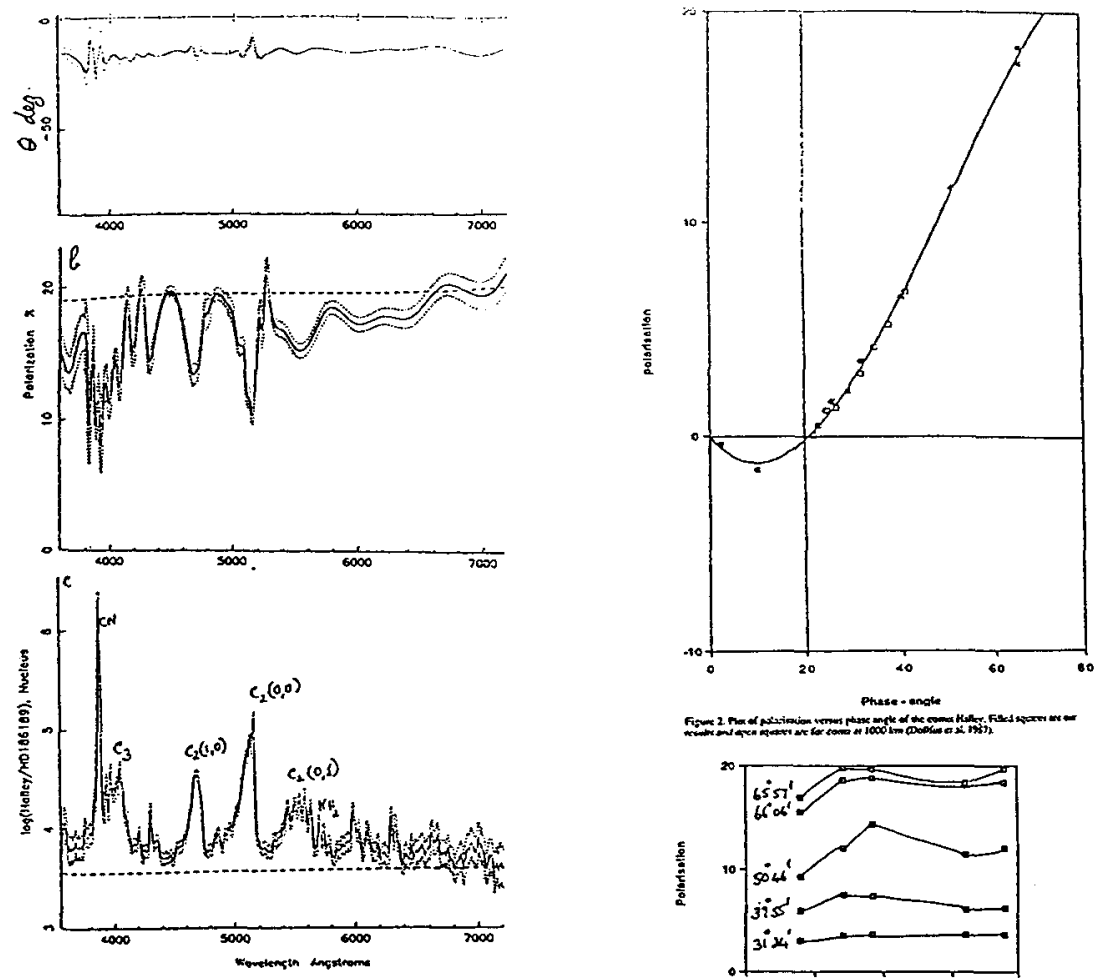

Figure 1 . The compulet poshion angle of the electric vector $\theta$ and polarization $P$ as a

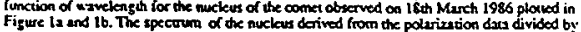

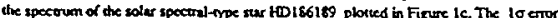
poines indieakd as doand tines. Main moloculer bunds marked.

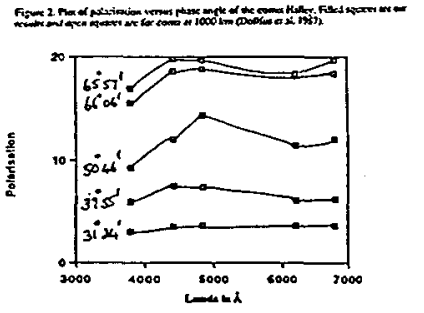

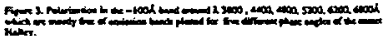

nucleus is in qualitative agreement with the results of other observers who used large size apertures (18-23 arcsec).

The mean polarization of the continuum of the nucleus of the comet at $\lambda 4800$ and $5200 \AA$ is computed for all the nine nights in Table 1 and plotted against the phase angle in Figure 2. It can be seen polarization becomes negative ( the angle $\theta$ is equal to the direction of the plane of scattering) for phase angles less than $20^{\circ}$. The maximum polarization $19.6 \%$ occurs at the phase angle $66^{\circ}$ and minimum polarization of $-2.0 \%$ at $\sim 10^{\circ}$. The relation of polarization with phase angle is nearly linear in the range 30 to $60^{\circ}$ with a cross-over at $21^{\circ}$ and a slope of $0.4316 \pm 0.07 \%$ per degree.Both pre-perihelion and post perihelion data form a continuous curve in Fig. 2. The data plotted in Fig. 2 represents the region around the nucleus of the comet with apertures $<1000 \mathrm{~km}$. Our data is in good agreement with the fresh dust polarization corrected - data at $1000 \mathrm{~km}$ in coma derived by Dollfus and Suchail (1987) during April 86 (Fig. 2). Though the minimum negative polarization and cross-over angle of our relation show agreement with other observers our slope of the phase-polarization relation is higher than that observed by other observers in the optical wavebands (Dollfus et al. 1988) and nearer to that observed at the IR (Brooks et al. 1987). This could be due to the fact that unlike the other data our data represents region of -2 arcsec around the nucleus which had a star-like appearance during 1985-86. Also it is possible that the polarization - correction for molecular emission applied by other observers may be in error by a few $\%$ especially for phase angles $>30^{\circ}$. The polarization in the second aperture which is 22.8 arcsec away in the sunward direction is smaller than that observed for the nucleus. 


\section{Interpretation}

Many models have been computed to interpret the polarization data of the reflected solar component from the comet. They require a mixture of rough silicate minerals and absorbing carbonaceous grains with a size distribution measured by space missions to Comet Halley (Lamy et al. 1987; Krishnasamy and Shah 1988 ). The negative part of the polarization - phase curve could be obtained by such a mixture. Albedo decreases with increase in size of the grains $(1-10 \mu \mathrm{m})$ from 0.035 to 0.02 which is in agreement with space data.

This low albedo of the Comet Halley is comparable to that of the rings of Uranus and the leading hemisphere of Iapetus some of the darkest material in the solar system. The maximum polarization and the negative part of the polarization - phase curve of the Comet Halley in Figure 2 are similar than those observed in asteroids (Dollfus 1989). This has been explained as Umov Effect which tells that the albedo $A$,and the maximum polarization $P_{\max }$ are related - when rough particles scatter, polarization increases with decreasing albedo. Thus again the $\mathrm{P}_{\max }$ in Figure 2 is consistent with low albedo of the comet particles. Using this comparison between the comet and the asteroids and the laboratory measurements of several meteorite lunar and other samples Dollfus (1989) finds that the optical polarization is matched by grains far larger than wavelength, very dark with an extremely rough surface formed as aggregates of small particles.

\section{References}

Brooke, T.Y., Knacke, R.F. and Joyce, R.R. (1987) 'The near-infrared polarization and color of comet P/Halley', Astron. Astrophys. 187, 621-624.

Dollfus ,A. and Suchail J.L.(1987) 'Polarimetry of grains in the coma of P/Halley', Astron. Astrophys. 187, 669-688.

Dollfus ,A.(1989)'Polarimetry of grains in the coma of P/Halley: II Interpretation ', Astron. Astrophys. 213, 469-478.

Dollfus ,A.,Bastien, P., Le Borgne, J.-F., Levasseur-Regourd, A.C., and Mukai,T. (1988)'Optical polarimetry of $\mathrm{P} / \mathrm{Halley}$ : synthesis of the measurements in the continuum', Astron. Astrophys. 206, 348-356.

Krishnasamy, K.S., and Shah, G.A.(1988)'Nature of dust grains in comets', M.N.R.A.S, 233, 573-579.

Lamy, P.L., Grun, E., and Perrin, J.M.(1987)'Comet P/Halley : implications of the mass distribution function for the photopolarimetric properties of the dust coma' , Astron. Astrophys. 187, 767-773.

McLean, I.S., Heathcote, S.R., Paterson, M.J., Fordham, J., and Shortridge, K. (1984), 'A multichannel Pockels cell spectropolarimeter for the Anglo-Australian Telescope'M.N.R.A.S, 209,655-664.

Visvanathan, N.(1965), 'Polarization in the Galaxy and the Large Magellanic Cloud' Ph.D. Dissertation, The Australian National University, pp. 68-70. 